\title{
A Review on the Relationship of Ergonomics and Industrial Hygiene
}

\author{
Hee-Sok Park \\ Department of Industrial Engineering, Hongik University, Seoul, 121-791
}

\begin{abstract}
Objective: The objective of this paper is to examine the scope of ergonomics in relation to the discipline of industrial hygiene. Background: There has been increasing recognition of the importance of ergonomics in the practice of industrial hygiene. As a result, quite a number of ergonomics-related research articles have been published in major industrial hygiene journals. And the professional engineers in industrial hygiene have conducted ergonomics-related activities. Method: This paper reports the results from the literature review of the ergonomics papers published in major industrial hygiene journals for the last 5 years, and the certification of industrial hygiene and ergonomics. Results: It was found that $7.7 \sim 13 \%$ of the total published papers were about ergonomics, and the number of the professional engineers in industrial hygiene is almost double of that of ergonomics. Conclusion: It should be understood that the work of ergonomic is complementary to industrial hygiene and not in competition with it. Application: Some suggestions were proposed hopefully helpful to colleague ergonomists for future research.
\end{abstract}

Keywords: Industrial hygiene, Complementary relationship

\section{Introduction}

최근에는 많은 학문분야가 학문 간의 경계가 없는 학제 적인 (interdisciplinary) 성격을 추구하고 있다, 인간공학도 마찬가지로서, 여러 학문분야에서 인간공학적 원리와 기 법을 연구 및 응용하고 있다. 예를 들어, 육체적 인간공학 (physical ergonomics)은 안전공학, 산업의학, 산업위생, 기 계공학 등의 분야에서, 그리고 인지적 인간공학(cognitive ergonomics)은 심리학, 산업디자인, 컴퓨터공학 등에서 관 심을 가지고 있다.

이러한 현상은 인간공학의 적용범위를 넓히고 학문의 위 상을 높이는 측면에서 바람직한 것으로 환영할 만 하다. 반 면, 인간공학 고유의 영역이 협소해지고 인간공학 전문가들 의 활동이 위축될 우려가 있다. 이에 본 논문에서는 인접학
문 중 국내외에서 인간공학 관련 연구 및 응용 활동을 활발 히 하고 있는 산업위생 (industrial hygiene) 분야와 인간공 학과의 관계를 짚어보고자 한다. 여기서 고려대상이 되는 인 간공학의 영역으로 인지적 인간공학 분야는 제외한다.

미국산업위생학회 (American Industrial Hygiene Association: AIHA) 는 산업위생을 '작업장에서 근로자에게 질병과 건강/복지의 손상을 유발하고 일반인의 불편함을 초 래하는 환경요인과 스트레스를 예측, 측정, 평가, 관리하는 과학과 기술이라고 광범위하게 정의하고 있다(AIHA Home page, 2011). 이 정의는 인간공학이 추구하는 바와 크게 다르지 않다. 또한 미국의 노동부 산하 산업안전보건청 (Occupational Safety and Health Administration: OSHA) 은 산업위생 분야가 다루어야 하는 위험요인으로서 공기 오 염물질과 화학적, 생물학적, 물리적, 인간공학적 요인을 거론 하고 있다(OSHA, 1998).

Corresponding Author: Hee-Sok Park. Department of Industrial Engineering, Hongik University, Seoul, 121-791.

Phone: 02-320-1473, E-mail: hspark@hongik.ac.kr

Copyright@2012 by Ergonomics Society of Korea(pISSN:1229-1684 eISSN:2093-8462). All right reserved.

(c) This is an open-access article distributed under the terms of the Creative Commons Attribution Non-Commercial License(http://creativecommons.org/licenses/by-nc/3.0/), which permits unrestricted non-commercial use, distribution, and reproduction in any medium, provided the original work is properly cited. 
그리고 주요 산업위생 전문학술지의 출판목적과 범위 (aims and scope) 가 인간공학을 포함하고 있어서 많은 인간 공학 관련 논문이 발표되고 있다. 또한 산업위생 자격인증의 출제범위에 인간공학이 포함되어 있으며, 산업현장에서도 산 업위생 전문가들이 인간공학적 응용 활동을 하고 있는 실정 이다. 더구나 최근 작업관련성 근골격계질환이 문제시 됨에 따라 많은 산업위생 종사자들이 인간공학에 대하여 관심을 가지고 있다. AIHA에서는 인간공학에 대한 입장(Position statement on ergonomics)을 통하여 근골격계질환의 평가 와 관리를 위한 인간공학의 역할과 범위를 정의하고, 관련 연구의 지원, 규제의 개발 등을 포함하는 AIHA의 역할을 명기한 바 있다(AIHA, 1997).

한편, 우리나라 정부에서는 근골격계질환의 업무상 질병 인정 여부를 결정함에 있어서 인간공학 전문가, 산업위생 전 문가, 산업의학 전문의 등 관련 전문가의 의견을 듣게 하고 있다(노동부 고시 제2008-43호). 즉, 근골격계질환의 공식 적인 인정에 있어 인간공학 전문가와 산업위생 전문가의 권 한이 동일한 것이다.

이렇듯 산업위생 분야에서는 인간공학을 구성 분야로 인 식하고 있으며, 활발한 인간공학 관련 연구와 응용 활동을 하고 있다.

이에 본 연구에서는 국내외 산업위생 전문학술지에 발표 되는 인간공학 관련 논문의 현황을 분석하여 산업위생 연구 계에서 차지하고 있는 인간공학의 비중을 살펴보았다. 그리 고 미국과 우리나라의 자격인증 제도의 현황을 조사하여 양 분야의 전문가 배출 정도를 비교하였다. 이를 통하여 산업위 생과 인간공학과의 관계와 인간공학 분야의 위상을 고찰하 고, 인간공학 연구자들이 지향해야 할 방향을 제시하고자 한다.

\section{Method}

\subsection{Research articles}

해외에서 발간되고 있는 주요 산업위생 전문학술지를 조 사하였다. 이를 위하여 Journal Citation Records에 수록 된 SCI등재학술지 중에서 제목에 hygiene이 포함된 것들 을 검색한 결과, 아래의 6종이 검색되었다: (1) American Journal of Tropical Medicine and Hygiene, (2) Annals of Occupational Hygiene, (3) Food Hygiene and Safety Science, (4) International Journal of Hygiene and Environmental Health, (5) Journal of Occupational and Environmental Hygiene, (6) Transactions of the Royal Society of Tropical Medicine and Hygiene. 이 중 산업위
생과 직접적인 관련이 없는 (1), (3), (6)번을 제외한 3개 학술지를 대상으로 하였으나, International Journal of Hygiene and Environmental Health는 그 범위에 인간공 학이 포함되지 않고 실제 인간공학 논문의 게재건수도 미미 하여 조사대상에서 제외하였다.

국내는 한국산업위생학회지 (한국연구재단 등재지, 년간 4호 발간)를 조사대상으로 하였다.

선정된 3개 학술지에 최근 5년간(2007 2011) 게재된 original article들을 위주로 분석하되, special issue는 제외 하였다.

육체적 인간공학과 환경 인간공학과 관련된 다양한 주제 어 (예: ergonomic, musculoskeletal, low back, intervention, risk factor, noise, hearing, vibration, heat, cold, lighting 등)를 사용하여 검색된 결과를 검토하였다. 그리고 게재된 모든 논문들의 제목과 초록을 검토하여 그 내용과 중심어가 상기한 기준에 해당되는 논문들을 추출하였다.

\subsection{Certification in the US and Korea}

미국에서 산업위생 전문자격을 부여하는 곳은 1960년에 설립된 American Board of Industrial Hygiene(ABIH)으 로서, Certified Industrial Hygienist $(\mathrm{CIH})$ 를 포함한 자격 을 부여하고 있다(ABIH Home page, 2011). 인간공학은 1990년에 설립된 Board of Certification in Professional Ergonomics (BCPE) 에서 수여하는 Certified Professional Ergonomist(CPE)를 조사하였다(BCPE Home page, 2011). 국내 자격제도는 한국산업인력관리공단에서 주관하는 산 업위생관리 기술사와 인간공학 기술사 제도를 중심으로 조 사하였다(HRD-Korea Home page, 2011).

\section{Results}

\subsection{Research articles}

Annals of Occupational Hygiene은 1958년부터 영국 산 업위생학회(British Occupational Hygiene Society)에서 발 간하여 왔으며, 2010년 기준 impact factor는 2.014이다. 년간 8호를 출간하고 있다. 최근 5 년간 게재된 총 논문 수는 379 편이며, 그 중 29 편 $(7.7 \%)$ 이 인간공학 관련 주제를 다 루는 논문이었다(Table 1).

Journal of Occupational and Environmental Hygiene은 AIHA와 산업위생 및 보건 관련 한계치(Threshold Limit Value: TLV)를 도출하는 미국정부산업위생가협회(American Conference of Governmental Industrial Hygienists: 
ACGIH)에서 공동으로 발간한다. 2010년 impact factor 는 1.293이다. 과거에는 다른 명칭 (AIHA Journal, Applied Occupational and Environmental Hygiene)이었으며, 2004 년부터 현재의 명칭을 갖는다. 매월 발간하고 있으며, 최근 5년 동안 게재된 총 417 편의 논문 중 48편(11.5\%)이 인간 공학 논문이었다(Table 2).

Table 1. The number of the articles published in the Annals of Occupational Hygiene

\begin{tabular}{c|c|c|c}
\hline Year & $\begin{array}{c}\text { Published } \\
\text { articles(T) }\end{array}$ & $\begin{array}{c}\text { Ergonomics } \\
\text { articles(E) }\end{array}$ & E/T(\%) \\
\hline 2007 & 67 & 5 & 7.5 \\
\hline 2008 & 72 & 2 & 2.8 \\
\hline 2009 & 76 & 6 & 7.9 \\
\hline 2010 & 81 & 9 & 11.1 \\
\hline 2011 & 83 & 7 & 8.4 \\
\hline Sum & 379 & 29 & 7.7 \\
\hline
\end{tabular}

Table 2. The number of the articles published in the Journal of Occupational and Environmental Hygien

\begin{tabular}{c|c|c|c}
\hline Year & $\begin{array}{c}\text { Published } \\
\text { articles(T) }\end{array}$ & $\begin{array}{c}\text { Ergonomics } \\
\text { articles(E) }\end{array}$ & E/T(\%) \\
\hline 2007 & 96 & 12 & 12.5 \\
\hline 2008 & 86 & 9 & 10.5 \\
\hline $2009^{*}$ & 75 & 5 & 6.7 \\
\hline 2010 & 81 & 13 & 16.0 \\
\hline 2011 & 79 & 9 & 11.4 \\
\hline Sum & 417 & 48 & 11.5 \\
\hline
\end{tabular}

*special issue 제외

한국산업위생학회지의 경우에는 인간공학 논문들이 전체 의 $13 \%$ 를 차지하고 있었다(Table 3 ).

Table 3. The number of the articles published in the Journal of Korea Society of Occupational and Environmental Hygiene

\begin{tabular}{c|c|c|c}
\hline Year & $\begin{array}{c}\text { Published } \\
\text { articles(T) }\end{array}$ & $\begin{array}{c}\text { Ergonomics } \\
\text { articles(E) }\end{array}$ & E/T(\%) \\
\hline 2007 & 36 & 4 & 11.1 \\
\hline 2008 & 34 & 5 & 14.7 \\
\hline 2009 & 36 & 8 & 22.2 \\
\hline 2010 & 32 & 2 & 6.3 \\
\hline $2011^{*}$ & 23 & 2 & 8.7 \\
\hline Sum & 161 & 21 & 13.0 \\
\hline
\end{tabular}

*2011년 12월 현재, 3호까지 발간

\subsection{Certification in the US and Korea}

미국 $\mathrm{CIH}$ 의 시험범위는 Air Sampling \& Instrumentation 을 비롯한 16 개 영역으로서, 인간공학이 명기되어 있다(시 간동작연구 포함). 현재 1 만 여명이 자격증을 보유하고 있 으며, 그 중 6,500 여명이 현직에서 활동하고 있다고 한다.

미국의 인간공학 기술사 자격증인 $\mathrm{CPE}$ 를 보유하고 있는 사람들은 따로 집계되어 있지 않으며, 약 1,500 여명이 다음 자격증을 보유하고 있다: Certified Professional Ergonomist, Certified Human Factors Professional, Certified User Experience Professional, Associate Ergonomics Professional, Associate Human Factors Professional, Associate User Experience Professional, Certified Ergonomics Associate.

국내의 산업위생관리 기술사는 1983년에 안전관리기술사 (산업위생관리)로 시작하여 1991년도에 산업위생관리 기술 사로 독립하였다. 시험문제 영역 중 산업위생학 개론(전체 시험문제 중에서 $20 \%$ )에 인간공학(들기 작업, 단순 및 반 복 작업, $\mathrm{VDT}$ 증후군, 노동생리, 근골격계질환, 작업부하 평 가방법, 작업환경의 개선) 과 산업피로(피로의 정의 및 종류, 원인 및 증상, 에너지 소비량, 작업강도, 작업시간과 휴식, 교대 작업, 산업피로의 예방과 대책) 부문이 인간공학과 직 접적으로 관련이 있다.

인간공학 기술사는 2005년부터 실시되고 있다.

양 기술사 시험의 지원자, 합격자 및 합격률 현황이 Table 4와 Table 5에 나타나 있다.

Table 4. The number of Professional Engineer in Industrial Hygiene Management

\begin{tabular}{c|c|r|r|r|r|c}
\hline \multirow{2}{*}{ Year } & \multicolumn{3}{|c|}{ Written test } & \multicolumn{3}{c}{ Oral test } \\
\cline { 2 - 7 } & Applicants & Pass & $\begin{array}{c}\text { Pass rate } \\
(\%)\end{array}$ & Applicants & Pass & $\begin{array}{c}\text { Pass rate } \\
(\%)\end{array}$ \\
\hline 2005 & 51 & 4 & 7.8 & 6 & 5 & 83.3 \\
\hline 2006 & 59 & 31 & 52.5 & 31 & 22 & 71 \\
\hline 2007 & 85 & 1 & 1.2 & 10 & 6 & 60 \\
\hline 2008 & 175 & 36 & 20.6 & 69 & 42 & 60.9 \\
\hline 2009 & 130 & 15 & 11.5 & 36 & 20 & 55.6 \\
\hline 2010 & 88 & 3 & 3.4 & 17 & 8 & 47.1 \\
\hline Sum & 588 & 90 & 15.3 & 169 & 103 & 60.9 \\
\hline
\end{tabular}


Table 5. The number of Professional Engineer in Ergonomics

\begin{tabular}{c|c|c|c|c|c|c}
\hline \multirow{2}{*}{ Year } & \multicolumn{3}{|c|}{ Written test } & \multicolumn{3}{c}{ Oral test } \\
\cline { 2 - 7 } & Applicants & Pass & $\begin{array}{c}\text { Pass rate } \\
(\%)\end{array}$ & Applicants & Pass & $\begin{array}{c}\text { Pass rate } \\
(\%)\end{array}$ \\
\hline 2005 & 49 & 14 & 28.6 & 14 & 8 & 57.1 \\
\hline 2006 & 38 & 19 & 50 & 25 & 12 & 48 \\
\hline 2007 & 27 & 9 & 33.3 & 22 & 8 & 36.4 \\
\hline 2008 & 15 & 5 & 33.3 & 16 & 8 & 50 \\
\hline 2009 & 20 & 9 & 45 & 11 & 5 & 45.5 \\
\hline 2010 & 18 & 1 & 5.6 & 7 & 4 & 57.1 \\
\hline Sum & 167 & 57 & 34.1 & 95 & 45 & 47.4 \\
\hline
\end{tabular}

\section{Conclusion and Discussion}

국내외에서 발간되는 산업위생 전문학술지를 조사한 결 과, 전체 논문의 7.7 13\% 정도가 인간공학 관련 논문이 었다. 산업위생의 다양한 분야를 고려하면 이는 결코 적은 비중이 아니라고 판단된다. 특히 AIHA에서는 1994년부터 'Ergonomics'라는 전문 칼럼을 신설, 운영하여 왔으며, 이 칼럼 이외에도 많은 인간공학 논문이 실리고 있다. 더구나 2000년도는 미국 내 인간공학 연구 및 규제, 예방관리의 괄 목할만한 해(banner year)로 기념하는 논문이 게재된 바 있 다(Schneider, 2001).

전문자격과 관련하여서는, 산업위생 분야의 오랜 역사로 인하여 산업위생 전문가들이 인간공학 기술사에 비해 수적 으로 2 배 이상 월등히 많은 것을 알 수 있었다. 국내 인간공 학 기술사의 활용방안으로 근골격계질환 예방관리 프로그램 구축, 유해요인 조사, 작업환경 개선이 가장 큰 비중을 차지 하는 것으로 조사되었으나(Lee, 2007), 이는 최근 산업위생 관리 기술사들이 참여하고 있는 영역이다. 특히 최근 국내에 서는 기술사 면접관 3 명중 1 명을 인간공학 전문가로 초빙하 는 등, 인간공학의 비중이 커지고 있다.

학술지에 발표되는 논문의 수와 전문자격 인증 현황만을 토대로 그 분야의 위상을 평가하는 것은 불충분하다. 그리고 논문의 분류가 주관적으로, 대분류만으로 이루어진 한계점이 있으나, 현재까지 인간공학 분야의 분류법(taxonomy)이 확 립되지 않은 실정임을 감안하여야 한다. 그리고 본 논문에서 채택된 방법 이외에도 해당 분야의 대학/대학원 학과 수 및 규모, 졸업생 수 등을 함께 분석한다면 보다 충분한 분석이 가능하겠다.

결론적으로, 산업위생 분야에서 인간공학 연구와 응용이
활발히 진행되고 있음을 알 수 있었다. 이러한 현상은 산업 안전, 산업의학도 비슷한 추세일 것이다. 그리고 육체적 인 간공학에 국한하여 고찰하였으나 인지적 인간공학도 같은 입장일 것으로 여겨진다.

서론에서 언급한 바와 같이 이러한 현상은 고무적이지만, 인접학문과 차별되는 인간공학의 정체성과 위상을 위하여 아래와 같이 제안하고자 한다.

(1) 여러 인간공학적 위험요인에 대한 양-반응 관계 (dose-response relationship)를 규명하는 보다 근원적인 연구가 필요하다. 현재 우리나라에서 적용되고 있는 인간공 학적 규제 또는 지침은 그 내용이 상당부분 정성적이며, 비 록 정량적이라 하더라도 논란이 많은 실정이다(예: 고용노동 부 지정 11 개 근골격계 부담 작업). 따라서 여러 인간공학 적 위험요인의 노출수준과 그 결과간의 정량적인 관계를 확 립하여 보다 과학적이며 노사가 모두 동의할 수 있는 합리 적인 규제를 도출할 필요가 있다.

(2) 외국에서 개발된 지침, 평가도구 또는 표준에 대한 우 리나라 집단에 대한 적합성을 검토해야 한다. 예를 들어, 들 기 작업에서의 적정하중 지침, 국제표준화기구(ISO)에서 제 정한 여러 인간공학 관련 표준 등이 우리나라 사람들에게 적 합한지를 검토하고, 필요한 경우 보정대책을 제시할 필요가 있다. 나아가, 서비스업, 비정형적인 작업 등 새로운 형태의 작업의 분석에 적합한 도구의 개발이 요구된다.

(3) 다른 공학분야에 대한 보다 높은 수준의 이해와 응용을 통하여 작업장의 공학적 개선 (engineering control) 조처를 도출할 필요가 있다. 최근에는 자동기계의 발전이 급속도로 이루어지고 있다. 이에 인간공학 연구자들은 인접한 공학분 야에 대한 관심을 통하여 보다 현실적이고 경제적인 개선안 도출을 주도할 필요가 있다. 아울러, 인간공학적 개선 프로 그램에 대한 경제성 분석을 함께 실시하여 인접학문과의 차 별화를 도모할 필요가 있다.

산업위생과 인간공학과의 관계는 경쟁적이지 않으며, 상호 보완적이다(Fraser, 1984). 상호에 대한 이해를 높이고 인 접학문의 접근방법을 서로 응용한다면 보다 폭넓은 연구가 가능하다(Schneider, 1997).

인간공학이 국내에 도입된 지 30여 년이 지났다. 그 동안 많은 노력을 통하여 양적, 질적 발전을 이루어왔다. 이제는 인접학문에 대한 이해를 높이고 융합적인 접근방법으로 광 범위하고 보다 심도 깊은 인간공학 연구와 응용이 필요한 시점이다. 


\section{Acknowledgements}

This work was supported by 2011 Hongik University Research Fund.

\section{References}

American Board of Industrial Hygiene Home page, http://www.abih.org (retrieved December 1, 2011)

American Industrial Hygiene Association, Position Statement on Ergonomics, AIHA Board of Directors, 1997.

American Industrial Hygiene Association Home page, http://www.aiha.org (retrieved December 1, 2011)

Board of Certification in Professional Ergonomics Home page, http:// www.bcpe.org(retrieved December 1, 2011)

Fraser, T. M., Ergonomics and Industrial Hygiene: A Complementary Relationship, American Industrial Hygiene Association Journal, 45(7), B5-B6, B8, 1984.

Human Resources Development Service of Korea Home page, http:// www.q-net.or.kr(retrieved December 1, 2011)

Journal of Korea Society of Occupational and Environmental Hygiene, Vol. 17-21, 2007 2011.

Lee, D., A Job Analysis of the Certified Professional Ergonomists, Journal of the Ergonomic Society of Korea, 27(2), 67-72, 2008.
Occupational Safety and Health Administration, OSHA 3143, Informational Booklet on Industrial Hygiene, U.S. Department of Labor, 1998.

Schneider, S. P., Applying Industrial Hygiene Principles to Control Ergonomic Risks, Applied Occupational and Environmental Hygiene, 11(10), 1190-1192, 1996.

Schneider, S. P., The Year 2000 in Ergonomics, Applied Occupational and Environmental Hygiene, 16(4), 432-437, 2001.

The Annals of Occupational Hygiene, Vol. 51-55, 2007-2011.

\section{Author listings}

Hee-Sok Park: hspark@hongik.ac.kr

Highest degree: $\mathrm{PhD}$, The University of Michigan

Position title: Professor, Department of Industrial Engineering, Hongik University

Areas of interest: Industrial Ergonomics, Musculoskeletal Disorders

Date Received : 2011-12-07

Date Revised :2012-01-10

Date Accepted : 2012-01-10 